\title{
NMR Study of the Morphology of Polystyrene Linear/Microgel Blends
}

\author{
K. J . McGrath ${ }^{\dagger}$ and C. M. Roland* \\ Chemistry Division, Code 6120, Naval Research Laboratory, Washington, D.C. 20375-5342
}

\section{Antonietti}

Max Planck Institute of Colloids and Interfaces, Kantstrasse 55, D-14513 Tel tow-Seehof, Germany

Received May 31, 2000

\begin{abstract}
The morphology of blends of polystyrene (PS) microgels with linear PS were studied using NMR spectroscopy. Three techniques were employed to assess the intimacy of mixing: isothermal crosspol arization/magic-angle-spinning (CP/MAS) dipolar dephasing ${ }^{13} \mathrm{C} N \mathrm{NR}$, temperature-dependent ${ }^{13} \mathrm{C} \mathrm{CP} /$ MAS NMR, and one-pulse ${ }^{1} \mathrm{H}$ MAS NMR. The experimental data reveal that linear PS can penetrate the core of soft microgels, whose network strands are substantially larger than the linear chains. However, when the microgel is highly cross-linked, such penetration is prevented. These results are in accord with Flory-Huggins swelling theory. Blends of linear PS with highly cross-linked microgels yield thermodynamically stable morphologies, in which the components are molecularly dispersed, yet segmental contacts are virtually absent. Such a phase morphology is novel and cannot be obtained with conventional polymer blends.
\end{abstract}

\section{Introduction}

Microgels. Polystyrene microgels are produced by simultaneous polymerization and cross-linking of monomers dispersed as an aqueous emulsion. ${ }^{1,2}$ The resulting particles (molecules) have the internal structure of a macroscopic network, yet there is negligible interpenetration among individual molecules. Near-neighbor contacts are al most exclusively intramol ecular, in contrast to conventional linear macromolecules. These particles are homogeneous, with a uniform segment density and lacking any surface "softness". ${ }^{3}$ The respective mass densities of microgels and linear PS are essentially the same, by virtue of the initially spherical microgel particles assuming a polyhedral form when coagulated to a neat melt. Of course, the size of an individual microgel particle is much less than the volume of a linear PS chain having the same molecular weight. The mean-square radius of gyration for linear PS is given by

$$
\left\langle\mathrm{R}_{\mathrm{G}}{ }^{2}\right\rangle=\frac{1}{6} \mathrm{C}_{\infty} \mathrm{nl}^{2}
$$

where $\mathrm{C}_{\infty}$ is the characteristic ratio $\left(=10.3\right.$ for $\left.\mathrm{PS}^{4}\right)$, and $\mathrm{n}$ and $\mathrm{I}(=1.54 \AA)$ are the number and length of backbone bonds, respectively. The corresponding quantity for microgel PS is

$$
\left\langle\mathrm{R}_{\mathrm{G}}{ }^{2}\right\rangle=0.29\left(\frac{\mathrm{M}}{\rho \mathrm{N}}\right)^{2 / 3}
$$

where $M$ is molecular weight, $\rho$ is the mass density $(=0.93 \mathrm{~g} / \mathrm{mL})$, and $\mathrm{N}$ is Avogadro's number. Thus, a million molecular weight microgel has a volume equal to that subtended by a linear PS chain of $M=50000$ Da.

The above considerations suggest the possibility of unique morphologies for blends containing microgels.

\footnotetext{
+ Current address: Department of Chemistry, Georgetown University, Washington, D.C. 20057.
}

This is especially true given the paucity of intermolecular contacts, which invariably preclude thermodynamic miscibility for conventional polymer blends in the absence of specific interactions. Polymer blending has long been regarded as a route to new properties without synthesis of new chemical structures. The unique structure of PS microgels-densely packed, unentangled polyhedrons-markedly affects their rheology and mechanical properties ${ }^{5-7}$ and offers the potential for interesting blend morphologies. Microgels have al so been proposed as model networks, offering a means to investigate the elastic properties of rubbery materials. ${ }^{3,8}$ Blends of linear chains with networks have been the focus of research into nematic coupling (i.e., orientational correlations) in deformed networks containing unattached probe chains ${ }^{9-14}$ and on the role of constraint release in the terminal relaxation of entangled polymers. ${ }^{15-17}$ In this paper we investigate PS microgels mixed with linear PS.

Blends of Linear Chains and Networks. The degree to which a network is swollen by linear chains reflects a balance between the excess entropy and the elasticity of the network chains. Generally, permeation of networks is expected to be limited, especially for long chains and high degrees of cross-linking. ${ }^{18-20}$ Indeed, most "semi-I PN" (interpenetrating polymer networks in which only one component is cross-linked) are phaseseparated materials. ${ }^{21}$ Semi-IPNs of cross-linked PS with chemically dissimilar linear polymers rely on specific interactions to achieve segmental-level mixing. ${ }^{22,23} \mathrm{An}$ additional problem with microgels is that their small size can restrict the extent of swelling, at least if there is a nonzero interfacial energy, to minimize this energy. 24,25

The swelling of PS microgels by small molecule solvents has been shown to vary with cross-link density in qualitative accord with theory and to be similar to results for conventional macroscopic PS networks. ${ }^{3}$ In the present experiments, the "solvent", linear PS, is polymeric. The Flory-Huggins expression, relating the volume fraction of the network at equilibrium swelling, 
$\phi$, to the interaction parameter, $\chi$, can be written 23,26

$$
\chi=\frac{1}{2 \phi \mathrm{N}_{\mathrm{x}}}-\frac{1}{\phi^{5 / 3} \mathrm{~N}_{\mathrm{x}}}-\frac{1}{\phi \mathrm{N}_{\mathrm{l}}}(1+\ln (1-\phi))
$$

in which $\mathrm{N}_{\mathrm{x}}$ is the number of chain units between crosslinks and $N_{\text {}}$ the degree of polymerization of the linear component. The first two terms of eq 3 represent the elastic energy of the network upon absorption of the linear polymer, while the last term is the entropy of mixing. The microgels per se are too large to directly contribute to the excess entropy. Equation 3 ignores any interfacial energy, which would also confer a dependence of the mixing thermodynamics on microgel size.

Assessment of Phase Morphology. The glass temperature of a miscible blend is expected to be intermediate between the $T_{g}$ 's of the neat components. ${ }^{27,28}$ Due to the ready availability of means to make such measurements, the observation of a single glass transition is the most common basis for assessing the phase morphology in a blend. For mixtures whose components have very different $T_{g}$ 's, the glass transition temperature of the mixture falls between those of the neat materials. NMR experiments have shown, however, that even when only a single glass transition is observed by bulk techniques such as DSC, motions of the components can occur at very different time scales. 29,30 Moreover, even phase-separated blends sometimes exhibit a single glass transition, since the resolution of methods such as DSC is limited in the detection of inhomogeneities. ${ }^{31}$

Microgel and linear PS have glass transition temperatures that are quite close. In such a circumstance, anomalies may arise, such as the blend transition not being intermediate between the respective $T_{g}$ 's of the neat components..$^{32-38}$ Generally, techniques relying on $\mathrm{T}_{\mathrm{g}}$ measurements are less informative for blends of nearly equal $T_{g}$. Herein we employ NMR to assess the intimacy of mixing. Previously we demonstrated the utility of ${ }^{129} \mathrm{Xe}$ NMR spectroscopy for probing the phase morphol ogy of blends. ${ }^{31-33} \mathrm{~A}$ variety of solid-state NMR techniques are available for investigating domain sizes and miscibility in polymers. These generally rely on observing some interaction between spins on different molecules or in different domains. To measure these interactions, spins in different environments must be differentiated, for example by isotopic substitution, 39,40 chemical shift, ${ }^{40-42}$ or differences in line widths. ${ }^{43-47}$

For spin- $1 / 2$ nuclei in solids (e.g., hydrogen and carbon), the dipole-dipole interaction provides a coupling that can give rise to spin diffusion or polarization transfer among nuclei. For the microgel blended with perdeuterated linear PS, we employ solid-state proton magic angle spinning 48,49 and rely on spin diffusion among hydrogen nuclei to determine the intimacy of mixing. Since the perdeuterated PS has approximately $1 \%$ residual hydrogen, a dipolar coupling, and associated spin diffusion, is possible between this residual hydrogen and the hydrogen of the microgel only if there is a homogeneous phase morphology.

Similarly, when a strong dipolar interaction between carbon and hydrogen nuclei exists, it is possible to transfer the nuclear polarization from the hydrogen to any carbon nuclei to which the former is coupled. In the present case, cross-polarization between the hydrogens of the microgel and the carbons of perdeuterated linear
Table 1. Polystyrenes

\begin{tabular}{|c|c|c|c|c|c|}
\hline PS & symbol & $\begin{array}{l}\text { cross-links } \\
\text { per chain } \\
\text { unit }\end{array}$ & $\begin{array}{c}M_{w} \\
(g / m o l)\end{array}$ & $\begin{array}{c}\left\langle\mathrm{R}_{\mathrm{G}}^{2}\right\rangle^{1 / 2} \\
(\AA)\end{array}$ & $\begin{array}{l}\mathrm{T}_{\mathrm{g}} \\
(\mathrm{K})\end{array}$ \\
\hline $\begin{array}{l}\text { deuterated linear } \\
\text { deuterated linear } \\
\text { protonated linear }\end{array}$ & $\begin{array}{l}\text { dl-9k } \\
\text { dl-29k } \\
\text { pl-50k }\end{array}$ & & $\begin{array}{l}8.8 \times 10^{3} \\
2.9 \times 10^{4} \\
5.0 \times 10^{4}\end{array}$ & $\begin{array}{l}8.8 \\
48 \\
63\end{array}$ & 356 \\
\hline $\begin{array}{l}\text { protonated } \\
\text { microgel }\end{array}$ & $\mu$ gel-10l & $1 / 10$ & $1.2 \times 10^{6}$ & 69 & 394 \\
\hline $\begin{array}{l}\text { protonated } \\
\text { microgel }\end{array}$ & $\mu$ gel-10h & $1 / 10$ & $5.0 \times 10^{7}$ & 240 & \\
\hline $\begin{array}{l}\text { protonated } \\
\text { microgel }\end{array}$ & $\mu$ gel-80 & $1 / 80$ & $1.2 \times 10^{6}$ & 61 & 378 \\
\hline
\end{tabular}

Table 2. Blends (Having Equal Weights of the Two Components)

\begin{tabular}{|c|c|c|c|c|c|}
\hline blend & $\begin{array}{l}\text { linear } \\
\text { PS }\end{array}$ & $\begin{array}{c}\text { linear } \\
\text { PS }\end{array}$ & $\begin{array}{l}\text { microgel } \\
\text { PS }\end{array}$ & mixing & $\mathrm{T}_{\mathrm{g}}(\mathrm{K})$ \\
\hline IA & dl-9k & & $\mu \mathrm{gel}-1$ & soluti & 366 \\
\hline IB & dl-9k & & $\mu \mathrm{gel}-10 \mathrm{~h}$ & solution & \\
\hline IC & dl-9k & & $\mu$ gel-80 & solution & 356 \\
\hline ID & dl-9k & & $\mu$ gel-10l & dry powder & 357,393 \\
\hline$I I A$ & dl-29k & pl-50k & & solution & \\
\hline
\end{tabular}

PS would be a consequence of a homogeneous phase morphology.

Thus, the proton MAS and carbon CP techniques are particularly appropriate in this investigation since both rely on the relative strength of dipolar couplings be tween protons of one component and either protons or carbon nuclei of the other. The first method demonstrates phase separation (miscibility) by an absence (presence) of strong hydrogen nuclear dipolar interactions at the residual hydrogens on the perdeuterated polymer. The second approach interprets CP between protons of the hydrogenous component with the carbons of the perdeuterated component as a manifestation of miscibility.

\section{Experimental Section}

The microgels were prepared by copolymerization of styrene and m-diisopropenylbenzene in water, using AIBN as the initiator.1,2 Mol ecular weights were determined by light scattering. The linear PS were obtained from Polymer Source, I nc. (Quebec, Canada). These materials are listed in Table 1. Mixtures were obtained by dissolution of the components in benzene, with subsequent vacuum-drying for 3 days at $50^{\circ} \mathrm{C}$. Additionally, physical blends (i.e., mixtures phase-separated by design) were prepared by manually grinding the powdered samples. All blends, whose designations are given in Table 2 , contained equal weights of the components.

Differential scanning calorimetry was carried out using a Perkin-EImer DSC-7 and Intracooler. Samples, whose weights varied between 4 and $8 \mathrm{mg}$, were cooled from $443 \mathrm{~K}$ at $10 \mathrm{deg} /$ min through $T_{g}$, with the latter taken as the midpoint of the exothermic shift.

NMR experiments were carried out on a Bruker DSX spectrometer with a static magnetic field of $7.04 \mathrm{~T}$, corre sponding to Larmor frequencies of 75.5 and $300.1 \mathrm{MHz}$ for ${ }^{13} \mathrm{C}$ and ${ }^{1} \mathrm{H}$, respectively. Hartmann-Hahn $\mathrm{CP}^{50-52}$ was employed using proton and carbon radio-frequency fields of $50 \mathrm{kHz}(5$ $\mu \mathrm{S} \pi / 2$ pulse width) and a MAS of $3.0 \mathrm{kHz} \pm 5 \mathrm{~Hz}$. NMR dipolar dephasing ${ }^{53-55}$ was used in conjunction with CP to rapidly dephase (broaden) resonances arising from carbons with directly bonded hydrogen, thus permitting observation of $\mathrm{CP}$ deuterated carbons. For these experiments, a fixed CP time of $6 \mathrm{~ms}$ was used, followed by a varying dipolar dephasing time $(0-100 \mu \mathrm{s})$. The resonances for the CP microgel carbons are rapidly dephased during the first $50 \mu \mathrm{s}$, whereby residual carbon intensity is due to deuterated linear PS carbon that is in segmental contact with the microgel. Thus, the absolute sensitivity of these experiments will be determined primarily 


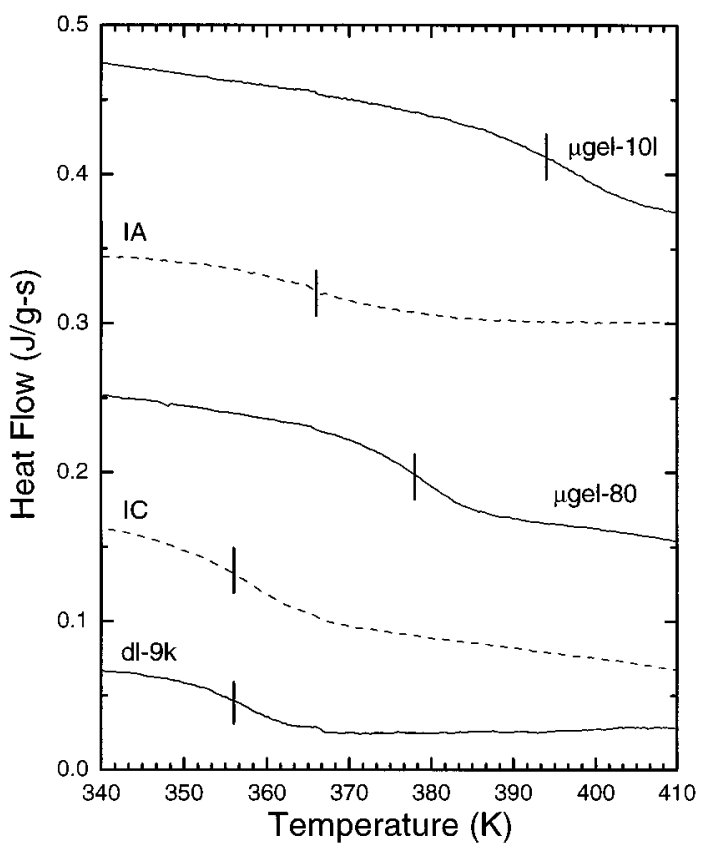

Figure 1. DSC cooling curves (10 deg/min), with the glass transition denoted by the vertical bar.

by the extent of dephasing that occurs for each dephasing time interval. Peak intensities among samples are then directly comparable, depending primarily on the strength of the proton-carbon dipolar interaction.

Temperature-dependent CP/MAS experiments were all obtained using a fixed $500 \mu \mathrm{s}$ CP time (no dipolar dephasing), whereby only microgel carbons are cross-polarized. The sensitivity of this experiment will vary from that obtained in the isothermal experiments (due to cross-polarization contact time difference, dipolar dephasing, and sample temperature), but the normalized peak intensities can once again be directly compared among samples.

Proton MAS experiments were carried out using a single 5 $\mu \mathrm{s} \pi / 2$ pulse, $3.0 \mathrm{kHz} \pm 5 \mathrm{~Hz}$ MAS, and a pulse repetition time of $20 \mathrm{~s}$. The ${ }^{1} \mathrm{H}$ spin-lattice $\left(\mathrm{T}_{1}\right)$ relaxation time constants were estimated to be $\leq 3 \mathrm{~s}$ for all samples, based upon separate experiments conducted on each of the neat samples.

\section{Results}

Differential Scanning Calorimetry. Displayed in Figure 1 are DSC results for two microgels, both neat and blended with the linear PS. Although all components are PS, the low molecular weight of the linear PS and the network structure of the microgel separates their Tg's to some extent.7,56 Note that each blend exhibits only a single transition. Blend IA has a $T_{g}$ falling between that of the components, $\mu \mathrm{gel}-10 \mathrm{l}$ and dl9k (see Tables 1 and 2). This is normal behavior.

On the other hand, blend IC, containing the less crosslinked microgel, exhibits a single $T_{g}$ at essentially the same temperature as the $\mathrm{T}_{\mathrm{g}}$ of the linear PS component. Note that this is the only transition observed in the blend. If the composition is present as a single phase, the heat capacity change occurring at the glass transition should be a simple mass-weighted average of the component heat capacity increments. Within the limited experimental precision of the data, this is indeed the case-the magnitude of the heat capacity change at $T_{g}$ for IC (as well as IA) is consistent with all the material present participating in the transition. Thus, the phase morphology is homogeneous on the length scale probed by calorimetry. The data in Figure 1, however, cannot distinguish between two possibilities: penetration of the linear PS into the microgel core (segmental mixing) versus a finely dispersed (even on the molecularly level) without such interpenetration. To probe the morphology further, we turn to NMR experiments.

Isothermal CP/MAS Dephasing ${ }^{13} \mathrm{C}$ NMR. The NMR technique of cross-polarization is a useful probe for intimacy of mixing, due to the strong dependence of the magnetization transfer rate on the internuclear separation. ${ }^{57,58}$ The inverse time constant for this process, i.e., the CP relaxation rate $T_{1 S^{-1}}$, is directly proportional to the second moment, $\mathrm{M}^{(2)} \mathrm{Sl}$, of the S spin (carbon) resonance line through dipolar coupling with the I spins (protons) ${ }^{57}$ for the high-effective-field, spinlock CP NMR experiment. The second moment is directly proportional to the inverse sixth power $\left(r^{-6}\right)$ of the distance separating the carbon and proton nuclei, ${ }^{59}$ and thus significant $\mathrm{CP}$ among ${ }^{13} \mathrm{C}$ and ${ }^{1} \mathrm{H}$ nuclei occurs only at distances less than $0.5-1.0 \mathrm{~nm} .60,61$

Following cross-polarization, it is possible to subject the carbon spins to a transient exposure to the proton dipolar interaction and thereby effect a dephasing, with consequent broadening and reduction of the carbon NMR resonance. Such a dipolar dephasing experiment ${ }^{53-55}$ is useful to probe miscibility since, given a fixed ${ }^{13} \mathrm{C}-{ }^{1} \mathrm{H}$ internuclear distance, the extent of the dephasing is proportional to the duration of the transient exposure of the carbon nuclei to the dipolar interaction of the protons; complete dephasing, or loss in carbon NMR resonance intensity, typically occurs in less than approximately $50-100 \mu \mathrm{s}$. In a miscible blend of perdeuterated linear PS and microgel, the carbons in the perdeuterated component which undergo CP from the hydrogens of the microgel will not undergo rapid dephasing due to the relatively large distance separating the deuterated carbons and the microgel hydrogens. This will result in a relatively high NMR resonance intensity from the deuterated carbons. In the case of an immiscible blend, all carbons that are cross-polarized will be rapidly dephased, since all of these carbons have directly bonded hydrogen. In the present experiments, the $\mathrm{CP}$ intensity of the deuterated carbons (following 50-100 $\mu$ s dipolar dephasing) reflects the relative quantity of perdeuterated linear PS that is intimately mixed (on the segmental level) with the second component (e.g., mi crogel). The aromatic carbon resonance of PS $(\delta=127 \mathrm{ppm})$ can be used to assess the extent of this cross-polarization.

Figure 2 is a plot of the carbon-13 NMR signal intensity at room temperature as a function of dipolar dephasing time (using a fixed CP time of $6 \mathrm{~ms}$ ) measured for blends containing the perdeuterated linear PS, dl-9k, with an equal weight of mi crogel (samples IA, IB, and IC). The figure also contains di polar dephasing data for perdeuterated and hydrogenous blends of two linear PS (IIA), as well as for a physical blend (ID) of dl-9k and $\mu$ gel-10h. The latter samples illustrate dephasing behavior for blends known to be miscible and phase segregated, respectively.

The linear PS mixture (II A) demonstrates that after 50-100 $\mu$ s di polar dephasing, approximately half of the CP aromatic carbon intensity remains, as expected in this miscible blend. ${ }^{62,63}$ Contrarily, in the physical blend (ID), in which intimate contact is precluded by the method of preparation, the aromatic carbon intensity falls off rapidly, due to the absence of cross-polarization of the deuterated carbons. They are all distant from the protonated species. 


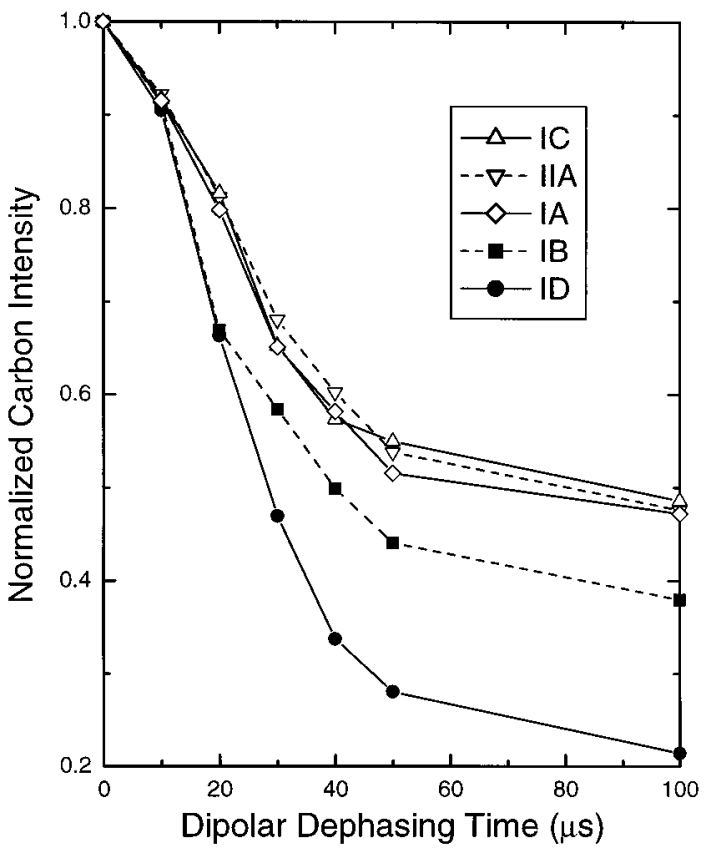

Figure 2. Normalized NMR aromatic carbon $(\delta=127 \mathrm{ppm})$ peak intensity in blends of dl-9k with microgels (IA, IB, IC, ID) and with a linear PS (IIA). A fixed CP contact time of 6 ms was used with varying dipolar dephasing times $(0-100 \mu \mathrm{s})$.

It is noted that for the physical mixture roughly $20 \%$ of the carbon intensity remains after $100 \mu \mathrm{s}$ of dipolar dephasing. This must reflect carbons from the deuterated component, cross-polarized by residual hydrogen during the $6 \mathrm{~ms}$ contact time. In fact, we observed similar intensities on neat samples of deuterated LPS and originally thought this reflected a high residual proton content. Liquid-state proton NMR experiments on these samples, however, confirmed that only $1-2 \%$ residual hydrogen was present. Possibly there is some contribution from RF inhomogeneity; nevertheless, the residual intensity from the perdeuterated component does not affect our qualitative conclusions from Figure 2 in any substantive way.

Figure 2 also reveals that for the two blends of microgel having lower molecular weight (i.e., I A and IC with $\mu$ gel-10l and $\mu$ gel-80, respectively) the CP/dipolar dephasing behavior is virtually identical to that of II A, indicating a substantial degree of intimate contact between the components. However, the CP/dipolar dephasing data behavior of the blend of linear PS with the higher molecular weight microgel (sample IB with $\mu$ gel-10h) is intermediate between that of the miscible and phase-segregated data. These results indicate that while there is molecular contact between the components, the segmental level intimacy is not necessarily identical for the three microgel blends.

It should be pointed out that the dipolar dephasing is not sufficiently short range to exclude surface contributions, and we expect that the outer few nanometers of a microgel particle will relax differently from its core. Hence, in principle, differing surface-to-volume ratios could also account for the observed size dependence. To resolve this ambiguity, we make use of temperaturedependent CP/MAS experiments to characterize the effect of mixing on the microgel's glass temperature.

Temperature Dependence of Efficiency of ${ }^{13} \mathrm{C}$ CP/MAS NMR. The results of the dephasing experiments may reflect linear PS that has diffused into the

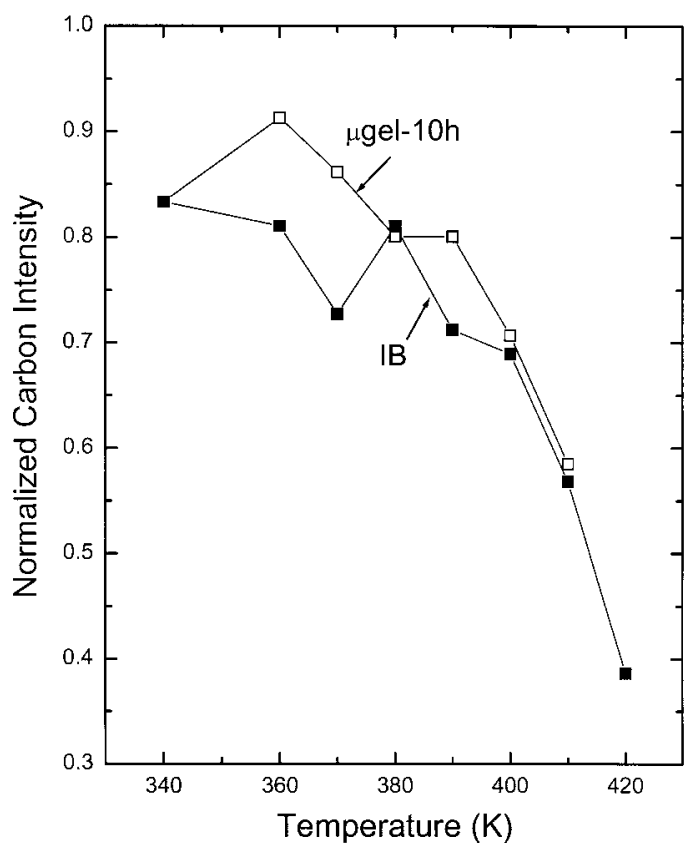

Figure 3. Normalized NMR aromatic carbon ( $\delta=127 \mathrm{ppm})$ peak intensity for $\mu$ gel-10h neat and blended with dl-9k. A fixed CP contact time of $500 \mu \mathrm{s}$ was used in order to polarize only the microgel carbons.

microgel core or that is only in contact with the microgel surface. Since the $T_{g}$ of the dll-9k is lower than that of the microgels, we expect some reduction in the glass temperature for the latter in those cases in which the linear PS has interpenetrated. To characterize this, we measure the efficiency of ${ }^{13} \mathrm{C} C P$ as a function of temperature.

Above $T_{g}$, the rate of segmental motion increases with increasing temperature. When the dynamics transpire on the time scale of the proton decoupling fiel $\mathrm{d}(50 \mathrm{kHz})$ or the MAS rate $(3 \mathrm{kHz})$, a reduction will be seen in the CP carbon resonance intensity. Additional loss of carbon intensity at elevated temperature will occur due to averaging of the nuclear dipole-dipole interaction between carbon and hydrogen, as well as due to the Boltzmann factor. Thus, by using a short CP contact time $(500 \mu \mathrm{s})$, whereby only microgel carbons become polarized, the effect of blending on the apparent glass temperature of the microgel alone can be determined from the CP (aromatic) carbon intensity's dependence on temperature.

Figure 3 shows the normalized, CP aromatic carbon intensity $(\delta=127 \mathrm{ppm})$ for $\mu \mathrm{gel}-10 \mathrm{~h}$ neat and blended (IB) over the temperature range 340-420 K. In both cases, the CP carbon intensity decreases with temperature, but surprisingly this occurs in similiar fashion over this range of temperatures. Thus, blending with low molecular weight PS has virtually no effect on the glass temperature of $\mu \mathrm{gel}-10 \mathrm{~h}$. Recalling that the results of Figure 2 indicate a level of segmental contact in this blend intermediate between fully miscible and phase segregated, we conclude that segmental contact must be confined primarily to the surface of the microgel, without actual penetration by the linear PS.

In Figure 4 we show the corresponding data for $\mu$ gel10l, again both neat and blended with linear PS (IA). As seen in Figure 3, the glass temperature of the microgel is unaffected by blending, consistent with an absence of chain interpenetration. However, unlike the 


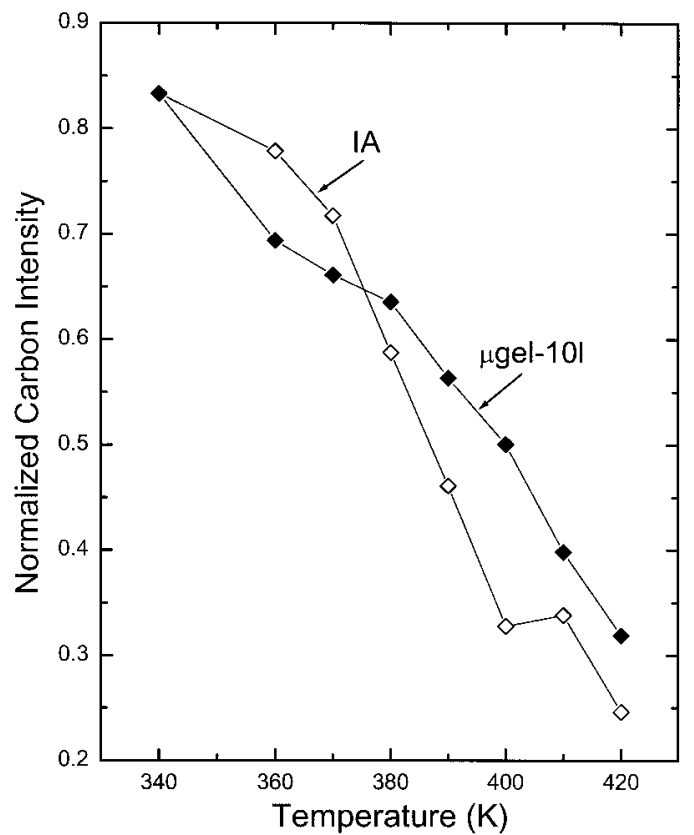

Figure 4. Normalized NMR aromatic carbon $(\delta=127 \mathrm{ppm})$ peak intensity in $\mu$ gel-10l neat and blended with dl-9k. A fixed CP contact time of $500 \mu \mathrm{s}$ was used in order to polarize only the microgel carbons.

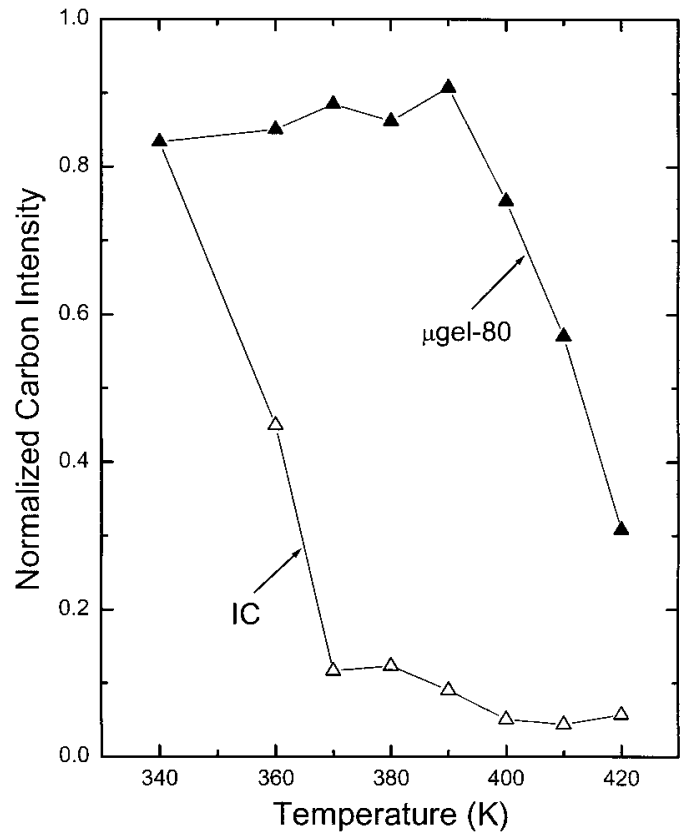

Figure 5. Normalized NMR aromatic carbon ( $\delta=127 \mathrm{ppm})$ peak intensity in neat $\mu$ gel-80 neat and blended with $\mathrm{dl}-9 \mathrm{k}$. A fixed CP contact time of $500 \mu \mathrm{s}$ was used in all experiments in order to polarize only the microgel carbons.

previous blend, the $\mathrm{CP} /$ dipolar dephasing results of Figure 2 indicate substantial segmental contact, roughly equivalent to that observed for the miscible blend of linear PS (IIA). The coil size for $\mu$ gel-10l is less than one-third that of $\mu$ gel-10h (Table 1), and thus the former has an order of magnitude greater surface area per unit mass. Evidently, the high degree of cross-polarization (Figure 2) for blend IA primarily reflects surface interaction. The 8K linear PS chains do not penetrate either microgel having $10 \%$ cross-linking.

In Figure 5 is shown the normalized aromatic carbon resonance intensities for the lower cross-linked microgel, $\mu$ gel-80, both neat and blended (IC). Unlike the results for $\mu \mathrm{gel}-10 \mathrm{l}$ and $\mu \mathrm{gel}-10 \mathrm{~h}$, in this case there is significant difference in behavior. While the neat microgel exhibits a reduction in carbon intensity with increasing temperature similar to that observed in Figures 3 and 4, for this blend the carbon intensity markedly decreases beginning around $350 \mathrm{~K}$. That is, $\mu \mathrm{gel}-80$ in blend IC has an NMR glass temperature approximately 40-50 deg lower than when neat. Obviously, this is a consequence of interpenetration by the lower $\mathrm{T}_{\mathrm{g}}$ dl-9k linear PS. Thus, the data in Figure 5 demonstrate intimate mixing (unlike the surface effects reflected in Figures 3 and 4) and resolve any ambiguity concerning surface contribution in Figure 2.

This effect of cross-link density on the penetration of the microgels is not surprising. Note that the radius of gyration of the network strands for the less cross-linked microgels is about $9 \AA$ (eq 1 ), roughly the size of the linear PS penetrant (dl-9k in Table 1). For $\mu$ gel-80, however, the network chains are 3 times larger than thelinear PS molecules, which enables interpenetration.

It is also noteworthy that the NMR gl ass temperature of $\mu$ gel-80 in blend IC appears to be lower than that of either of the neat components comprising this mixture. Such anomalous behavior has been observed previously in several miscible blends, $30,32,34-37,64-66$ and can arise from various factors. Herein we can conclude at a minimum that, unlike the blends of linear PS with the more cross-linked microgel, blend IC is molecularly dispersed.

One-Pulse ${ }^{1} \mathbf{H}$ MAS NMR. NMR line width and peak intensity measurements for proton resonances reflect the magnetic environment of the hydrogen atoms. The residual hydrogens in the perdeuterated linear PS (dl9k and dk-29k) are isolated magnetically from other protons, and thus yield narrow peaks $\left(\delta v_{1 / 2} \approx 1 \mathrm{kHz}\right.$ when observed using $3 \mathrm{kHz}$ MAS), compared to protons on hydrogenous PS ( $\delta v_{1 / 2} \approx 30 \mathrm{kHz}$ for $3 \mathrm{kHz}$ MAS). In addition, unlike carbon-13 CP measurements, the integrated intensity of the hydrogen peak will be directly proportional to the number of hydrogen atoms present in the sample, since the experiments were repeated on a time scale that is long compared with the ${ }^{1} \mathrm{H}$ spinlattice relaxation times.

Figure 6 displays the mass-normalized, integrated proton intensities for blends IB and IC (dl-9k mixed with $\mu$ gel-10h and with $\mu$ gel- 80 , respectively), al ong with data for the physical blend (ID) and the linear PS blend (IIA). For the physical blend, the relatively narrow aromatic and aliphatic MAS peak intensities are governed primarily by magnetic dipole-dipole interactions among residual hydrogens in the perdeuterated component. In particular, the aromatic peak intensity is quite low. Blend IB, for which Figure 3 revealed minimal penetration of the microgel by the linear PS, likewise exhibits low aromatic intensity. However, this aromatic intensity is substantially larger for the two miscible blends, IIA (the linear PS mixture) and IC (viz. Figure 5). The increase in the aromatic intensity reflects the strong interaction among the phenyl groups. Dimer formation between benzene rings is well-known, ${ }^{67-69}$ even leading to aggregation phenomena in aromatic polymers. ${ }^{70,71}$ Phenyl protons in proximity to a deuterated benzene ring will be in a diluted proton environment, contributing to a greater proton peak intensity in Figure 5 . That is, their weaker proton-proton dipolar interaction will be more effectively averaged by MAS, narrowing the 


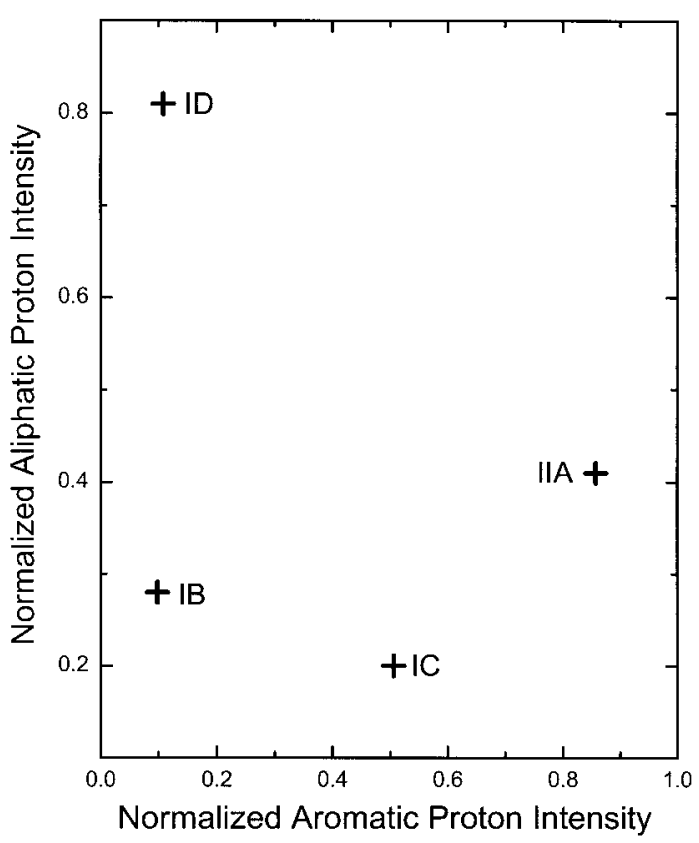

Figure 6. Normalized aromatic vs aliphatic proton NMR peak intensity data for dl-9k blended with various microgels. The relatively high aromatic proton peak intensity in blends IC and II A is a consequence of the dilution of phenyl hydrogens in the protonated microgel component by the perdeuterated linear PS.

Table 3. Phase Morphology

\begin{tabular}{ccc}
\hline blend & NMR results & $1-\varphi$ (eq 3) (\%) \\
\hline IA & interfacial contact & 4 \\
IB & interfacial contact & 4 \\
IC & microgel penetration & 24
\end{tabular}

proton peak widths to yield higher peak intensities. Thus, the position along the abscissa scale in Figure 5 directly reflects the intimacy of the mixing.

In contrast to the data for the aromatic resonances, all solution-blended samples, whether intimately mixed (IC and II A) or lacking intermolecular penetration (IB), have low aliphatic intensities, unlike the phase-separated, physical mixture (ID). Evidently, these intensities are decreased due to broadening, which arises from interaction of the residual protons on the dl-9k with the hydrogens of the microgel. As a consequence, the aliphatic hydrogen peak intensity is not directly dependent on the intimacy of contact between the components in these blends.

\section{Discussion and Summary}

The results herein demonstrate that the compliance of microgels allows probe molecules, including polymer chains, to enter their core. However, high cross-link density inhibits this penetration. These results, which are summarized in Table 3, can be compared to predictions from eq 3 . We calculate the fraction of linear chains within the microgel particle (i.e., $1-\phi$ ), with the assumption that the mixture is athermal $(\chi=0)$. If fact, this overestimates the miscibility, since the presence of the cross-link junctions, which have a different chemical structure, will reduce compatibility. ${ }^{72} \mathrm{~F}$ or the microgels, the cross-link unit (diisopropenylbenzene) differs only modestly from the styrene monomer, so that we expect a significant contribution to the thermodynamics only in blends of IA and IB, in which cross-link junctions are $10 \%$ of the total.
Nevertheless, eq 3 still predicts a 6-fold higher concentration of linear PS in the less cross-linked microgel (Table 3). If the effect of the cross-link junction on $\chi$ were included, the calculated miscibility of the blends with the $10 \%$ cross-linked mi crogel will be even less. Note also that increasing the particle size of the microgels (viz. blend IB in comparison to IA) does not promote miscibility, in accord with a low interfacial energy, due to the chemical similarity of the components. Thus, the results of the NMR experimental results are qualitatively consistent with theory.

For tightly cross-linked microgels, specific interactions between the probe and the microgel $(\chi<0)$ are required to achieve mixing on the segmental level. Yet even when penetration of the microgel is precluded, a molecular dispersion of the linear chains and the microgel is obtained. Such a morphology is quite distinct from conventional polymer blends, which the components are either phase-separated on a macroscopic scale $\left(\geq 10^{-7}\right.$ $\mathrm{m}$ ) or dispersed on the segmental (subchain) level. The novel morphology obtainable with microgels opens up the possibilities of both interesting new physics and new physical properties.

Acknowledgment. The work at NRL was supported by the Office of Naval Research. C. A. Aronson contributed to the DSC experiments.

\section{References and Notes}

(1) Antoniettii, M.; Basten, R.; Lohmann, S. Macromol. Chem. Phys. 1995, 196, 441.

(2) Antonietti, M.; Bremser, W.; Muschenborn, D.; Rosenauer, C.; Schupp, B. Macromolecules 1991, 24, 6636.

(3) Antonietti, M.; Bremser, W.; Schmidt, M. Macromolecules 1990, 23, 3796.

(4) Graessley, W. W.; Edwards, S. F. Polymer 1981, 22, 1329.

(5) Antonietti, M.; Pakula, T.; Bremser, W. Macromol ecules 1995, 28, 4227.

(6) Roland, C. M.; Bero, C. A.; Ngai, K. L.; Antonietti, M. Mater. Res. Soc. Proc. Ser. 1996, 411, 367.

(7) Roland, C. M.; Santangelo, P. G.; Antonietti. M.; Neese, M. Macromolecules 1999, 32, 2283.

(8) Antonietti, M. Macromol. Symp. 1995, 93, 213.

(9) Queslel, J.-P.; Erman, B.; Monnerie, L. Polymer 1988, 29, 1818.

(10) J acobi, M. M.; Stadler, R.; Gronski, W. Macromol ecules 1986, 19,2884

(11) Sotta, P.; Deloche, B.; Herz, J .; Lapp, A.; Durand, D.; Rabadeux, J .-C. Macromol ecules 1987, 20, 2769.

(12) Kornfield, J. A.; Fuller, G. G.; Pearson, D. S. Macromol ecules $1989,22,1334$

(13) Tassin, J. F.; Bashwitz, A.; Moise, J. Y.; Monnerie, L. Macromolecules 1990, 23, 1879.

(14) Zemel, I. S.; Roland, C. M. Polymer 1992, 33, 4522.

(15) Nelb, G. W.; Pedersen, S.; Taylor, C. R.; Ferry, J . D. J . Polym. Sci., Polym. Phys. Ed. 1980, 18, 645.

(16) Adachi, K.; Kotaka, T. Prog. Polym. Sci. 1993, 18, 585.

(17) Schroeder, M. J .; Roland, C. M. Macromolecules, 1999, 32, 2000.

(18) Bastide, J .; Candau, S.; Leibler, L. Macromol ecules 1981, 14, 719.

(19) Adachi, K.; Nakamoto, T.; Kotaka, T. Macromolecules 1989, $22,3106$.

(20) Wu, W.-L.; Wallace, W. E.; van Zanten, J . H.; Bauer, B. J .; Liu, D.-W.; Wong, A. Polymer 1997, 38, 2583.

(21) Coleman, M. M.; Serman, C.J .; Painter, P. C. Macromolecules 1987, 20, 226.

(22) Frisch, H. L.; Klempner, D.; Yoon, H. K.; Frisch, K. C. Macromolecules 1980, 13, 1016.

(23) Bauer, B. J .; Briber, R. M.; Han, C. C. Macromolecules 1989, $22,940$.

(24) Gilbert, R. G. Emulsion Polymerization: A Mechanistic Approach; Academic Press: New York, 1995; p 57.

(25) Antonietti, M.; Kaspar, H.; Tauer, K. Langmuir 1996, 12, 6211.

(26) Flory, P. J .; Rehner, J . J . Chem. Phys. 1943, 11, 521. 
(27) Roland, C. M. In Handbook of Elastomers-New Devel opments And Technology; Bhowmick, A. K., Stephens, H. L., Eds.; Marcel Dekker: New York, 1988; Chapter 6

(28) Roland, C. M. Rubber Chem. Technol. 1989, 62, 456.

(29) Lohfink, M.; Sillescu, H. In Proceedings of 1st Tohwa University International Symposium; Kawasaki, K., Kawakatsu, T., Tokuyama, M., Eds.; American Institute of Physics: New York, 1992.

(30) Roland, C. M.; Santangelo, P. G.; Ngai, K. L.; Meier, G. Macromol ecules 1993, 26, 6164.

(31) Walton, J. H.; Miller, J. B.; Roland, C. M.; Nagode, J . M. Macromol ecules 1993, 26, 4052.

(32) McGrath, K. J .; Roland, C. M. Rubber Chem. Technol. 1994, $67,629$.

(33) Walton, J. H.; Miller, J. B.; Roland, C. M. J . Polym. Sci., Polym. Phys. Ed. 1992, 30, 527.

(34) Roland, C. M. Macromolecules 1995, 28, 3463.

(35) Roland, C. M.; Santangelo, P. G.; Baram, Z.; Runt, J . Macromol ecules 1994, 27, 5382.

(36) Fried, J. R.; Karasz, F. E.; MacKnight, W. J . Macromolecules 1978, 11, 150

(37) McGrath, K.J .; Roland, C. M. Macromol ecules 1995, 28, 2982.

(38) Fujita, H. Fortschr. Hochpolym.-Forsch. 1961, 3, 1.

(39) Schaefer, J .; Sefcik, M. D.; Stejkal, E. O.; McKay, R. A. Macromol ecules 1981, 14, 188.

(40) Vander Hart, D. L.; Manders, W. F.; Stein, R. S.; Herman, W. Macromolecules 1987, 20, 1724.

(41) Caravatti, P.; Neuenschwander, P.; Ernst, R. R. Macromolecules 1985, 18, 119.

(42) Caravatti, P.; Neuenschwander, P.; Ernst, R. R. Macromolecules 1986, 19, 1889.

(43) Miller, J. B.; McGrath, K. J .; Roland, C. M.; Trask, C. A.; Garroway, A. N. Macromol ecules 1990, 23, 4543.

(44) McGrath, K.J .; Ngai, K. L.; Roland, C. M. Macromolecules 1992, 25, 4911.

(45) McGrath, K. J .; Ngai K. L.; Roland, C. M. Macromolecules 1995, 28, 2825.

(46) Goldman, M.; Shen, L. Phys. Rev. 1966, 144, 321.

(47) Landry, C. J . T.; Henrichs, P. M. Macromolecules 1989, 22 2157.

(48) Andrew, E. R. Arch. Sci., Geneve 1959, 12, 103.
(49) Lowe, I. J . Phys. Rev. Lett. 1959, 2, 285

(50) Hartmann, S. R.; Hahn, E. L. Phys. Rev. 1962, 128, 2042.

(51) Pines, A.; Gibby, M. G.; Waugh, J. S. J . Chem. Phys. 1973 $59,569$.

(52) Schaefer, J .; Stejskal, E. O. J . Am. Chem. Soc. 1976, 98, 1031.

(53) Opella, S.J .; Frey, M. H. J . Am. Chem. Soc. 1983, 105, 2142

(54) Roland, C. M.; Miller, J. B.; McGrath, K. J . Macromolecules 1993, 26, 4967.

(55) McGrath, K. J .; Roland, C. M. Macromolecules 1992, 25, 166

(56) Santangelo, P. G.; Roland, C. M. Macromolecules 1998, 31, 4581.

(57) Demco, D. E.; Tegenfeldt, J .; Waugh, J . S. Phys. Rev. B 1975, 11,4133

(58) Levitt, M. H.; Suter, D.; Ernst, R. R. J . Chem. Phys. 1986 , $84,4243$.

(59) Slichter, C. P. Principles of Magnetic Resonance, 2nd ed. Springer: New York, 1980; Vol. 1.

(60) Douwel, C. H. K.; Maas, W. E. J . R.; Veeman, W. S.; Buning, G. H. W.; Vankan, J . M. J . Macromolecules 1990, 23, 406.

(61) Parmer, J . F.; Dickinson, L. C.; Chien, J . C. W.; Porter, R. S. Macromolecules 1987, 20, 2308.

(62) McGrath, K. J .; Roland, C. M.; Weiss, R. G. Macromolecules 1993, 26, 6127.

(63) McGrath, K.J .; Roland, C. M. Macromolecules 1992, 25, 1366

(64) Alegria, A.; Telleria, I.; Colmenero, J . J . Non-Cryst. Solids 1994, 172, 961.

(65) Santangelo, P. G.; Ngai, K. L.; Roland, C. M. Macromolecules 1994, 27, 3859

(66) Santangelo, P. G.; Roland, C. M.; Ngai, K. L.; Rizos, A. K.; Katerinopoulos, H. J . Non-Cryst. Solids 1994, 172-174, 1084

(67) Basile, L. J . J . Chem. Phys. 1962, 36, 2204.

(68) Vala, M. T.; Haebig, J.; Rice, S. A. J . Chem. Phys. 1965, 45, 886.

(69) Nishihava, T.; Kaneko, M. Makromol. Chem. 1969, 124, 84

(70) Sonnenschein, M. F.; Roland, C. M. Polymer 1990, 31, 2023.

(71) Sonnenschein, M. F.; Roland, C. M. J . Polym. Sci., Polym. Phys. Ed. 1991, 29, 431.

(72) Brochard, F. J . Phys. (Paris) 1981, 42, 505.

MA000951X 